\title{
Autosurfactant treatment of acute postoperative respiratory failure
}

\author{
Mustafin A., Mustafin A.,_Sagimbayev A., Medical University Astana, \\ Dept of Anaesthesiology \& Intensive Care, Astana, Kazakhstan
}

\section{Background:}

Autosurfactant replacement therapy is used by us to increase the extensibility of the lung parenchyma. In his introduction took place a clear reduction of the aerodynamic resistance of the airways and increase in tidal volume.

\section{Materials and Method:}

Autosurfactant therapy was performed in 20 patients with clinical signs of acute postoperative respiratory failure. Bronchoalveolar lavage (BAL) was obtained from healthy subsegmental bronchi of the lungs, before surgery in an amount of $40-50 \mathrm{ml}$., it was filtered through a layer of sterile gauze, centrifuged at low temperature for 10 minutes. The supernatant was used for the study of surfaceactive properties (PAS) of the alveolar surfactant and therapy autosurfactant. Surface Tension (PN) BAL examined by the Wilhelmy scales. In addition, the bronchoalveolar washings examined the content of total lipids, phospholipids, protein and albumin. For the treatment of acute postoperative respiratory failure autosurfactant used with good surface-active properties, where PN minimum was $24+-3.0 \mathrm{mN} / \mathrm{m}$.

\section{Results:}

After introduction autosurfactant there was an increase in tidal volume by $10.3 \%$, decreased aerodynamic drag as you exhale $11.8 \%$, increased lung complains $21.4 \%$. These changes of respiratory function were accompanied by a decrease in work of breathing to overcome the elastic resistance of the lungs. The bronchoalveolar washings obtained from healthy lung areas before surgery, lung surfactant was kept at its optimum biochemical composition, which has good adsorption and distribution, efficient clearance mechanism phospholipids as it is the patient's own surfactant. There are practically no negative qualities of exogenous surfactant. We also took into account that the lavage fluid, according to the literature, the optimal therapy. Autosurfactant replacement therapy is used by us to increase the extensibility of the lung parenchyma. In his introduction took place a clear reduction of the aerodynamic resistance of the airways and increase in tidal volume.

\section{Conclusion:}

Autosurfactant therapy may prevent the development of ARDS in the development of acute postoperative respiratory failure in the immediate period after surgical. Exogenous surfactant may cause allergic reactions. However autosurfactant not antigenic properties with respect to the recipient and can not cause allergic reactions, because of their biochemical composition, it is the same as in the recipient. 